\title{
Wireless Data Acquisition in Flight Test Networks
}

\author{
Diarmuid Collins \\ Curtiss-Wright \\ Corporation \\ Dublin, Ireland
}

\begin{abstract}
The use of wireless data networks is ubiquitous in the consumer world. They have gained significant traction due to advantages afforded by the lack of wires. These same advantages can prove valuable in Flight Test for data acquisition. Sensor nodes are ideal candidates for low bandwidth wireless networks. Located in remote, hard to reach and hostile environments, wirelessly acquiring data from such sensor can solve a number of existing issues for FTI engineers. Implementing such wireless communication introduces a number of challenges such as guaranteeing reliable transfer of the sensor data and time synchronization of the remote nodes. This paper addresses wireless sensor acquisition, the associated challenges and discusses approaches and solutions to these problems.
\end{abstract}

Keywords: WSN, Wireless Sensor Networks, LXRS, Wireless LAN

\section{INTRODUCTION}

Flight Test Instrumentation (FTI) systems extensively utilize wired networking technologies because they are a proven technology; however, in a number of scenarios wired networks present significant shortcomings. Most of these shortcomings, ironically, relate to the unavoidable fact that the networks involve wires.

One of the most significant of these limitations is accessibility, in a number of scenarios it is simply not possible to route a wire to a remote sensor thereby making acquisition impossible. Another limitation is weight. As networks increase in size and complexity, the weight of the wires contributes to a significant proportion of the total weight of the acquisition system. Additionally, the inflexibility of a wired network is another aspect which limits the utility of such a system and can significantly increase the cost of testing.

Wireless networks alleviate many of these issues. The ability of the wireless network to easily and quickly change its topology and augment an already installed network is a significant advantage that wireless brings. This paper will examine these issues and will propose solutions based on wireless technology. In particular, solutions involving wireless sensor networks will be addressed.

\section{WIRELESS DATA ACQUISITION SCENARIOS}

There are a number of specific use cases that can be considered for wireless data acquisition. Addressing these scenarios can help to identify the particular requirements for each scenario and therefore help in selecting the most suitable technology. The following 
are a list of uses cases identified in FTI

\subsection{USE CASES}

1. Wireless Sensors: Remote sensors gather measurements and the data is transmitted to a wireless data acquisition user module that allows the measurements to be transmitted on the wired network

2. Point-to-point Wireless Data Acquisition: Data acquired from various sensors and busses is acquired in a wireless remote DAU. The acquired data is then transmitted wirelessly to a receiving wireless DAU.

3. Wireless Bridge. Two wired Ethernet networks can be bridged using a wireless link. This allows two networks which may be physically impossible to connect, be bridged using a wireless link.

4. Wireless Access Point: Multiple remote wireless RDAUS can associate with an Access Point and transmit the acquired data wirelessly to the access point. An access point is typically a router and a wireless bridge in the one unit. This unit also allows wireless clients such as real time analysis PCs connect to the FTI network.

5. Wireless Data Mining: A PC with a wireless network card can connect to the FTI Figure 1 Wireless Acquisition Scenarios network to mine the data recorded from the acquisition system.

6. Telemetry: A long-range Ethernet link to the ground to allow part of the acquired data be transmitted to the ground as well as commands sent to the FTI network.

\subsection{FTI SPECIFIC CHALLENGES}

Wireless networks in commercial spaces have already addressed a number of the issues which wireless communications systems encounter. Network contention is primary among these. Many protocols use a Carrier Sense Multiple Access with Collision Avoidance (CSMA/AC) schemes as a channel access method. This mechanism introduces packet latency, packet loss and a reduction in the transmit determinism and still may suffer collisions due to the hidden terminal problem or simultaneous transmission. These are all undesirable factors in FTI networks, where guaranteed delivery of packets, is a strict requirement. As a result, mechanisms to mitigate against packet loss should be accounted for in FTI wireless networks.

The variability in the packet delay through the network has a significant impact on time synchronization mechanisms such as Precision Time Protocol (PTP) [1]. PTP is a widely used time synchronization mechanism in FTI networks, allowing remote data acquisition units to be synchronized accurately to a common time source. In replacing wired links with wireless connections, the time synchronization of remote DAUs need to be maintained or replaced with equivalent schemes. 


\section{WIRELESS TECHNOLOGIES}

There are a number of wireless standards that are currently in use in the commercial world. To match the FTI scenarios previously identified to wireless standards, it's important to compare these standards, identifying advantages and disadvantages.

\subsection{WIRELESS LAN}

IEEE 802.11 is a set of standards that operate primarily in the $2.4 \mathrm{GHz}$ and $5 \mathrm{GHz}$ bands. This standard is commonly known as Wi-Fi, and widely used in the office and at home. There are a number of protocols defined using this standard, currently the most widely used being $802.11 \mathrm{~g}$ and $802.11 \mathrm{n}$. The key features are briefly summarized here

\begin{tabular}{|l|l|l|l|l|}
\hline & $\mathbf{8 0 2 . 1 1 b}$ & $\mathbf{8 0 2 . 1 1 g}$ & $\mathbf{8 0 2 . 1 1 n}$ & $\mathbf{8 0 2 . 1 1 a c}$ \\
\hline Release & 1999 & 2003 & 2009 & 2014 \\
\hline $\begin{array}{l}\text { Frequency } \\
\text { (GHz) }\end{array}$ & 2.4 & 2.4 & $2.4 / 5$ & 5 \\
\hline $\begin{array}{l}\text { Max Data } \\
\text { Rate } \\
\text { (Mbit/s) }\end{array}$ & 11 & 54 & 150 & $1000+$ \\
\hline $\begin{array}{l}\text { Modulation } \\
\text { DSSS }\end{array}$ & $\begin{array}{l}\text { DSSS, } \\
\text { OFDM }\end{array}$ & OFDM & OFDM \\
\hline $\begin{array}{l}\text { Range } \\
\text { (LOS m) }\end{array}$ & 100 & 100 & 250 & \\
\hline
\end{tabular}

Figure 2 Wi-Fi Standards

The key features that Wi-Fi brings are relatively high data rates and wide adoption in the commercial world. The data rates approach or exceed 100Base-T Ethernet rates, making it suitable for scenarios where significant data is transmitted such as a Wireless Network bridging and Wireless Access Points. However with such data rates, come significant power requirements. A single chip WLAN module could easily draw up to $1000 \mathrm{~mW}$ when transmitting at $802.11 \mathrm{~g}$ data rates. [2] For devices line-powered this could be acceptable but for battery powered devices which need to remain autonomous, this is quite high power consumption. For wireless sensor networks, the combination of high data rates and high power consumption is not ideal.

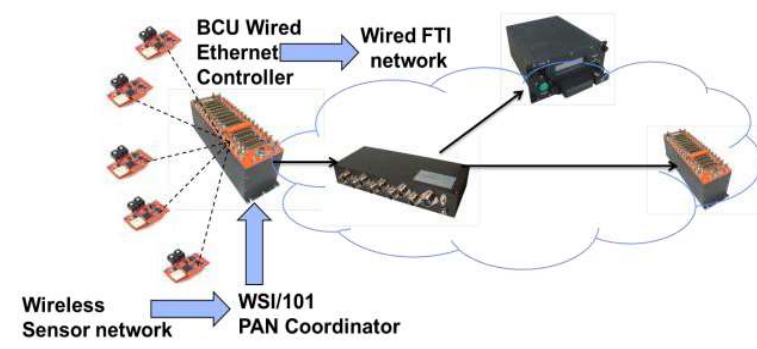

Figure 3 Zigbee Acquisition Module

\subsection{ZIGBEE}

Zigbee is a networking standard generally used for low data rate, home automation and industrial control applications such as lighting control. It is based on the IEEE 802.15.4 radio standard and supports applications that require periodic short data transfers up to $250 \mathrm{kbit} / \mathrm{s}$ over distances to $75 \mathrm{~m}$. The standard provides for low end-to-end latency and is a 
relatively mature standard. However there are still a number of shortcomings in the standard that has been explored in previous papers and experimental developments. [3] Contention when accessing the network has a significant impact on the aggregate data rate. Time synchronization of the remote Zigbee nodes is also not part of the standard, whereas accurate timestamping of wireless samples in FTI is crucial. Zigbee provides a very interesting option for Wireless Sensor Networks while not having the complete answer.

\subsection{BLUETOOTH}

Bluetooth is another wireless standard for use over short distances. It's was originally standardized as IEEE 802.15.1 but is now maintained by the Bluetooth SIG. Data is transmitted using a Time Division mechanism which makes is suitable for FTI wireless sensor networks. Bluetooth is generally considered a short-range communication protocol which is typically less than 10 meters. However the radio uses a frequency-hopping spread spectrum mechanism, which can complicate regulatory compliance in a tightly regulated environment on a plane.

\subsection{ANT +}

ANT is a proprietary wireless technology developed by Dynastream Innovations. It's a targeted at low data rates using a TDM system in the $2.4 \mathrm{GHz}$ spectrum. The data rates supported are quite low ( 20kbps) which limit the application area.

\section{WIRELESS SENSOR NETWORKS}

A Wireless Sensor Network (WSN) is network of spatially distributed sensors that monitor physical or environmental conditions such as temperature, pressure, strain, etc. These wireless sensors transmit the acquired data to an acquisition system. Wireless sensors are typically small with ultra-low power requirements running on batteries or utilizing energy harvesting schemes.

The ease of installation of wireless sensor networks brings advantages to debug or temporary installs. The install time for a wireless sensor network is quicker than a wired network. Furthermore, modification of an existing sensor network is significantly quicker than modification of wired installs. This has been demonstrated in a Cabin Comfort install on the A350 MSN2. [4]

\subsection{DATA SOURCES}

Typically sensors are sampling analog data sources with varying profiles of data rates.

- Acceleration. Typical accelerometers contain multiple channels to measure on three planes of motion. Sampling rates vary but can reach rates of $\mathrm{kHz}$. As a result they can be quite demanding on bandwidth.

- Temperature. In many scenarios, temperature changes are relatively slow allowing low sampling rates.

- Strain. Similar to accelerometers, strain measurements range from very low sampling rates measure in samples per hour to hundreds of $\mathrm{Hz}$.

In many of these measurements the acquisition systems follow a similar approach. The data is sampled, some signal conditioning is performed

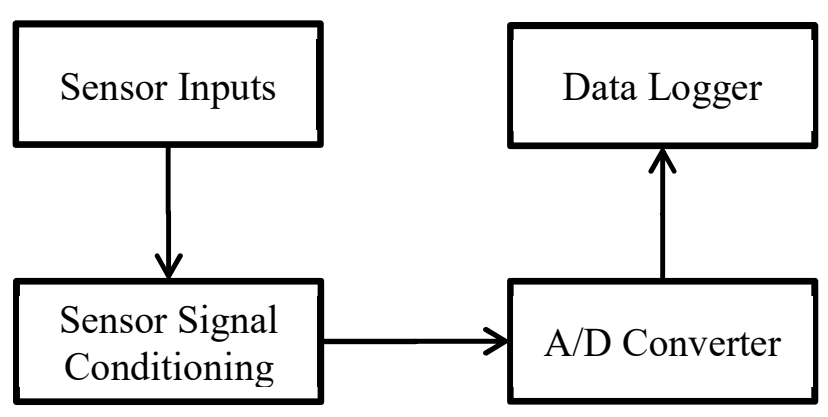

Figure 4 Signal Acquisition 
and the resulting data is logged. The signal conditioning can vary between applications and installations so should be programmable.

\subsection{LXRS}

As previously mentioned there are a number of wireless protocols that can be used for wireless sensor networks, however many come with limitations. An alternate approach is to use a proprietary wireless protocol that specifically addresses these limitations. LXRS is a wireless communication protocol which is designed for (but not limited to) the IEEE 802.15.4 communication standard. LXRS describes a method for guaranteed data delivery, mitigation of channel contention, and measurement synchronization. All of which, are essential features of a wireless measurement system..

\subsubsection{Medium Access Control}

Data transfer using the LXRS protocol follows a Time Division Multiplexed (TDM) approach. The bandwidth on the network is divided into time slots. Each sensor node on the network is programmed to transmit only during the assigned time slot. Multiple time slots can be assigned to a node to meet the aggregate data rate requirements of the node. This approach removes the impact of collisions to the data transfer, increasing the determinism and network throughput while significantly reducing the number of retransmits.

\subsubsection{Time Synchronization}

In FTI networks, the accuracy of timestamping of analog samples is a key requirement. The ability to synchronize and align samples taken from a wireless sensor with samples from standard wired sensors is crucial in the analysis of the acquired data. To achieve this on the wireless sensor

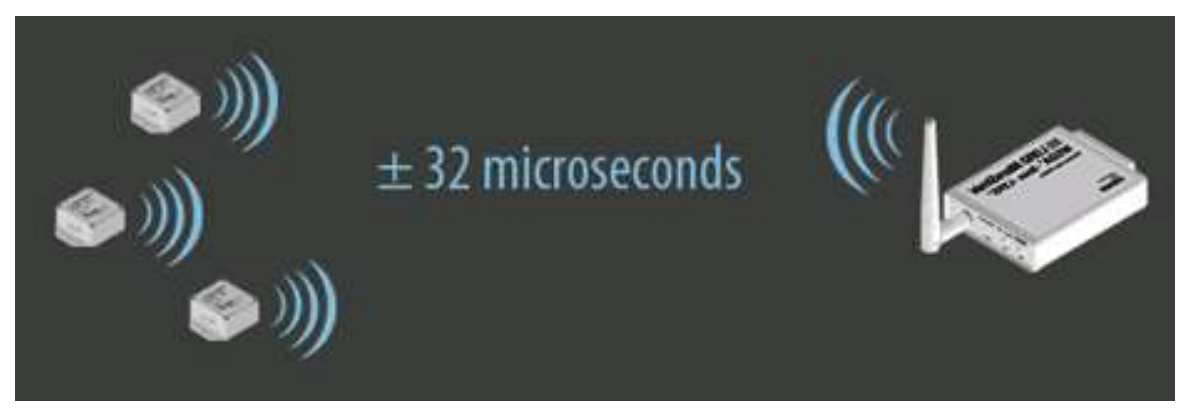

from the wireless gateway. The wireless sensors use high precision real-time clocks to maintain time stability between beacon re-synchronizations which occur every 20 seconds. In a setup where the Wireless gateway is integrated into a wired Ethernet network, the source time could eventually come from a PTP grandmaster.

\subsubsection{Data Integrity}

While implementing a TDM based data transfer mechanism reduces packet collisions between wireless nodes, due to the nature of wireless networks, elimination of all packet loss is still a significant challenge. The channel environment itself posts a number of challenges which a communication system needs to overcome. Specifically, due to a changing environment with 
multiple transmission paths, the wireless signal can suffer serious degradation which varies over time. Sensors can be moved or physical obstructions can temporarily interfere with transmit and receive paths. Temporary radio interference from other transmission sources may also cause packet loss.

LXRS includes a mechanism for overcoming the packet loss due to these temporary interference scenarios. Packets which are successfully received by the wireless gateway are acknowledged in a handshaking mechanism. Each wireless sensor contains some non-volatile memory in which the wireless samples are stored. Packets that are not acknowledged remain within NVM on the node and are scheduled for retransmission at later time. The time-stamping of the sample is also recorded so that the timestamp matches the sampling time and not the transmit time, meaning that the timing fidelity of the measurement is maintained despite being buffered.

\subsubsection{Extended Range}

WSN which been designed to utilize LXRS have also been designed to support a larger transmit range. The transmit range is a function of the transmit power and the nature of the environment. In a line of sight application the range is between $1.5 \mathrm{~km}$ and $2 \mathrm{~km}$ depending on the regulatory requirements. In more confined and congested environments the effective range is reduced. Additionally, in harsh RF environments characterized by severe frequency selective fading, the increased transmit power serves to increase the reliability of the RF link by increasing the link budget.

\subsection{POWER CONSUMPTION AND SENSOR AUTONOMY}

The ideal wireless sensor will consume no power, allowing the sensor to remain installed with no power source or requirement to change batteries. However for the sensor to measure, process or transmit, some power will be consumed. Minimizing this power consumption will extend the time between either replacement of the battery or recharging of the battery.

The operation of the wireless sensor can be split into three distinct modes of operation

- $\quad$ Logging and processing of sensed data. Typically $5 \mathrm{~mW}$.

- Wireless transmission of sensed data. Typically $45 \mathrm{~mW}$.

- $\quad$ Sleeping between data samples. Typically $0.02 \mathrm{~mW}$ [5]

To maximize battery life of the remote sensor each mode of operation should be optimized. However it is clear from the figures that limiting the wireless transmission operations would have the largest impact on the battery life. By locally logging the sensed data in the wireless sensor and then transmitting a burst of samples would be a more efficient use of the battery rather than transmitting each sample. LXRS supports such a mechanism [6].

Judiciously configuring the remote sensor to sample at the minimum rate needed to meet the measurement requirement would be the next approach in maximizing battery life. With two orders of magnitude between sleep mode and logging of data, it is clear that only sampling data at the minimum required rate will maximize battery life.

\subsubsection{Energy Harvesting}


While the ideal wireless sensor consuming no power is not achievable, an alternate solution is to use an energy harvesting mechanism on the sensor. This would provide for a fully autonomous sensor, removing the requirement for battery replacement or recharging. However the efficacy of such a device is highly depended on the amount of non-electrical energy (vibration, thermal gradients, cyclical strain, etc...) found within the environment. Each harvester requires a significant level of customization to operate within specific scenarios.

Due to the power consumption of the measurement and transmission system, this facility is best suited to low sampling rate measurements like strain or temperature. At $10 \mathrm{~Hz}$ it has been shown than power consumption of a wireless sensor node can be implemented at $90 \mu \mathrm{W}$ [7]. This level of power can be satisfied by piezoelectric energy harvesters or photovoltaic sources.

Higher samples rates have been achieved in a number of applications including monitoring the loads on pitch-links continuously at $128 \mathrm{~Hz}$ [8] and rotor system vibration periodically at 4 kHz. [9]

\subsection{INTEGRATION IN ACQUISITION SYSTEMS}

The Wireless Sensor Network, once acquiring data from remote sensors, needs to be integrated into the same acquisition system as all the wired sensors, forming a homogeneous network.

This includes:

- Programming the WSN using standard network protocols such as TFTP and SNMP.

- Transmitting acquired data using the same packet formats, for example IENA or iNet-X.

- Time synchronization using PTP or NTP.

With modern open standards such as XidML [10], it is possible to store information on how data is acquired, processed and transmitted in an FTI network. When used in combination with configuration software such as DASStudio [11], it is possible to configure and acquire data from WSN, just as easily as from wired sensors.

Once recorded, the source of parameters, whether wired or wireless, is transparent to the FTI engineer analyzing the results. This makes it even easier to move between wired and wireless sensors.

\section{CONCLUSION}

It is trivial to imagine a number of scenarios in which wireless technology can solve existing FTI problems or make existing solutions more flexible, efficient and cheaper. However the introduction of wireless technologies will not be without its challenges. In Wireless Sensor Networks, reliable transmission of acquired data and time synchronization issues present challenges which can be addressed using wireless standards such as LXRS. Integration of such protocols into existing network infrastructure is possible by using acquisition modules like the Curtiss-Wright KAM-500 module, the KAM/WSI/104. The gains that wireless sensor networks promise provide an attractive goal for the move to wireless.

\section{REFERENCES}

[1] N. Cranley and D. Corry, "Analysis and Application Scenarios for Telemetry Data 
Transmission and Synchronization over Wireless LAN," in ITC, 2008.

[2] Redpine, "RS9110-N-11-03 802.11abgn WLAN Module Data Sheet," 2012. [Online]. Available: $\quad$ http://www.redpinesignals.com/pdfs/RS9110-N-11-03/RS9110-N-1103_Module_DataSheet_v1.31.pdf.

[3] N. Cranley, "Experimental Investigation of Wireless Technologies for Data Acquisition," ITC.

[4] J. Galibert, A. Plo and S. Garay, "Cabin Comfort Flight Tests Installation," ETTC, 2015.

[5] S. Arms, C. Townsend, J. Galbreath and A. Newhard, "Wireless Strain Sensing Networks," in European Workshop on Structural Health Monitoring, Munich, 2004.

[6] LORD MicroStrain, "LXRS Data Communications Protocol," 9 July 2013. [Online]. Available: $\quad$ http://files.microstrain.com/Wireless-Sensor-Networks-LXRS-DataCommunication-Protocol.pdf.

[7] Microstrain, "Power Management for Energy Harvesting Wireless Sensors," 2005.

[8] S. Arms, "Tracking Pitch Link Dynamic Loads with Energy Harvesting Wireless Sensors," 2007.

[Online]

Available: http://www.microstrain.com/sites/default/files/applications/files/Wireless-Energy-HarvestingPitch-Link-Load-Monitoring.pdf..

[9] D. Wells, "Development of a Helicopter On-Rotor HUM System Powered by Vibration Energy Harvesting," 40th European Rotor Forum (ERF2014), 2014.

[10] "XidML," [Online]. Available: http://www.xidml.org/.

[11] Curtiss-Wright, "DAS Studio 3," 2015. [Online]. Available: http://www.cwcae.com/product/das-studio-3. 